PREPARED FOR THE U.S. DEPARTMENT OF ENERGY, UNDER CONTRACT DE-AC02-76-CHO-3073

PPPL-3007

PPPL-3007

, UC-420,427

TEMPERATURE ANISOTROPY IN A CYCLOTRON RESONANCE HEATED TOKAMAK PLASMA AND THE GENERATION OF POLOIDAL ELECTRIC FIELD

BY

W. CHOE, C.S. CHANG AND M. ONO

NOVEMBER 1994

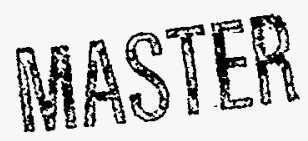

DISTRIBUTION OF THIS DOCUMENT IS UNLIMITED

YP

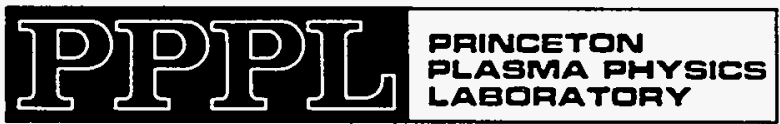

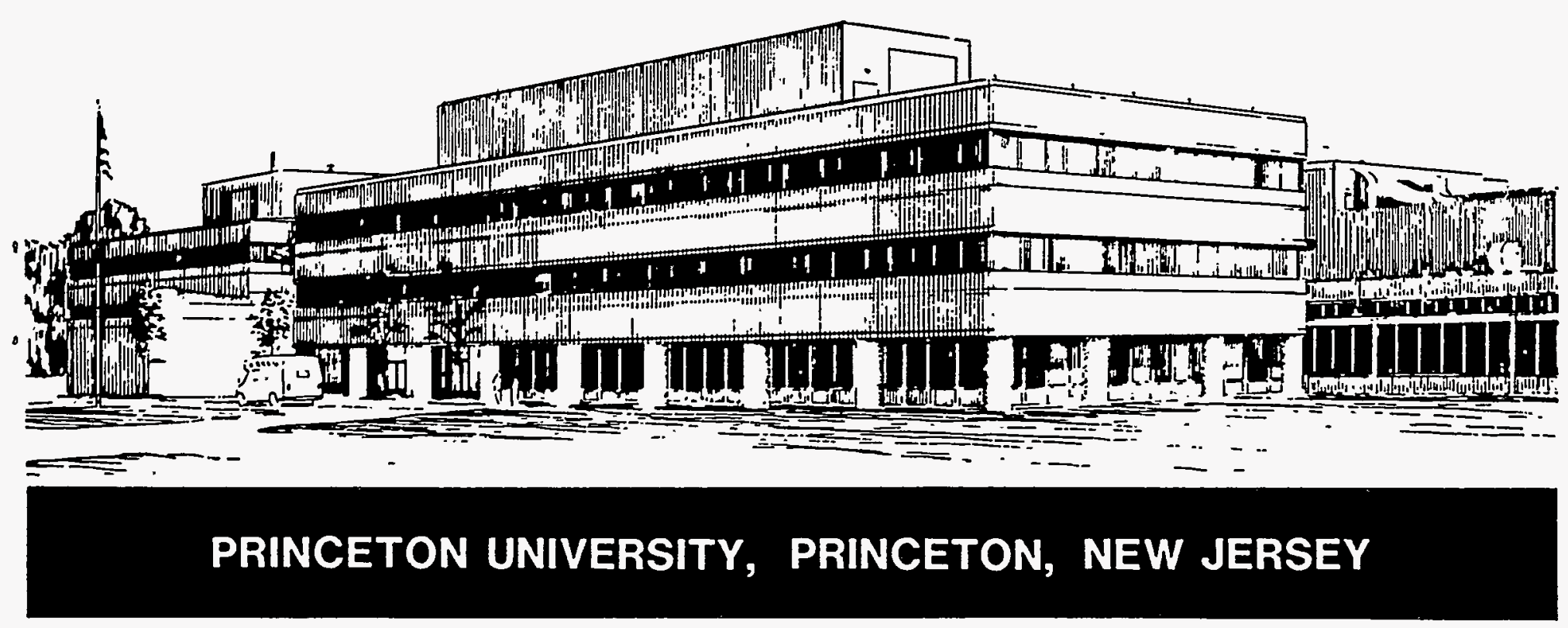




\section{NOTICE}

This report was prepared as an account of work sponsored by an agency of the United States Government. Neither the United States Government nor any agency thereof, nor any of their employees, makes any warranty, express or implied, or assumes any legal liability or responsibility for the accuracy, completeness, or usefulness of any information, apparatus, product, or process disclosed, or represents that its use would not infringe privately owned rights. Reference herein to any specific commercial produce, process, or service by trade-name, trademark, manufacturer, or otherwise, does not necessarily constitute or imply its endorsement, recommendation, or favoring by the United States Government or any agency thereof. The views and opinions of authors expressed herein do not necessarily state or reflect those of the United States Government or any agency thereof.

\section{NOTICE}

This report has been reproduced from the best available copy. Available in paper copy and microfiche.

Number of pages in this report: 37

DOE and DOE contractors can obtain copies of this report from:

Office of Scientific and Technical Information

P.O. Box 62

Oak Ridge, TN 37831 ;

(615) 576-8401.

This report is publicly available from the:

National Technical Information Service

Department of Commerce

5285 Port Royal Road

Springfield, Virginia 22161

(703) $487-4650$ 


\section{DISCLAIMER}

Portions of this document may be illegible in electronic image products. Images are produced from the best available original document. 


\title{
Temperature anisotropy in a cyclotron resonance heated tokamak plasma and the generation of poloidal electric field
}

\author{
W. Choe, C.S. Chang*, and M. Ono \\ Princeton Plasma Physics Laboratory, Princeton University \\ P.O. Box 451, Princeton, NJ 08543 \\ ${ }^{*}$ Courant Institute of Mathematical Sciences \\ New York University, 251 Mercer Street, New York \\ New York 10012
}

The temperature anisotropy generated by cyclotron resonance heating of tokamak plasmas is calculated and the poloidal equilibrium electric field due to the anisotropy is studied. For the calculation of anisotropic temperatures, bounce-averaged FokkerPlanck equation with a bi-Maxwellian distribution function of heated particles is solved, assuming a moderate wave power and a constant quasilinear cyclotron resonance diffusion coefficient. The poloidal electrostatic potential variation is found to be proportional to the particle density and the degree of temperature anisotropy of warm species created by cyclotron resonance heating.

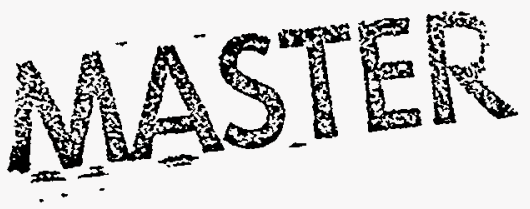




\section{INTRODUCTION}

Ion or electron cyclotron resonance heating is known to preferentially increase the perpendicular energy of resonant particles so that the distribution function of the heated particles is no longer isotropic. The increase of the perpendicular energy leads to more banana trapping at the outboard side of a tokamak where the magnetic field strength is weak. This will cause an imbalance of spatial charge on a magnetic flux surface. In order to maintain charge neutrality, an electrostatic potential with an in-out poloidal asymmetry is created. Under certain circumstances, the magnitude of this potential variation can be significant.

The equilibrium poloidal electric field in a tokamak plasma was usually neglected in the early neoclassical theories $[1,2]$ based on the fact that any space charge on a magnetic surface can be short-circuited by fast moving electrons. Leaving alone the case of cyclotron resonance heating, the existence of poloidal electric fields has been suggested to arise in the presence of high- $Z$ impurity species in ohmically heated plasmas [3,4], or due to the high collisionality in the plasma edge region [5]. Both parallel and perpendicular neutral beam injection [6] were also suggested to give rise to these potentials.

In this paper, we calculate the anisotropic temperatures of cyclotron resonance heated particles with a bi-Maxwellian model of warm particle distribution, and we discuss the generation of poloidal electrostatic potential variation due to temperature anisotropy. In the case of electron cyclotron resonance heating, an excessive number of electrons are trapped at the outboard bound of toroidal plasma. Thus, the electrostatic potential will be lower at the outside than the inside. On the other hand, ion cyclotron resonance heating can lead to higher electrostatic potential at the outside.

Similar calculations of the poloidal potential variation by electron cyclotron resonance heating can be found elsewhere [7-9]. Hsu et al. [7] assumed zero parallel 
wavenumber, non-relativistic, and a large aspect ratio, and they included only pitch angle scattering for the Coulomb collision term in the low collisionality regime. They showed an $\epsilon$-dependence of the potential. Chan et al. [8] included Doppler effect in the quasilinear term and calculated the poloidal electric field by an adjoint method. They concluded that the ratio of the potential energy to the kinetic energy is proportional to $\epsilon$ but its magnitude is much smaller than $\epsilon$ in a collision dominated plasma. Taguchi et al. [9] also used the adjoint method along with an eigenfunction technique in the banana regime. They assumed low-beta tokamak with circular cross-section and weak wave power $\left(\frac{\nu_{R F}}{\nu_{e e}} \ll 1\right)$, and included Doppler effect and relativistic correction to the resonance condition. They showed that the magnitude of the potential is proportional to $\frac{\nu_{R F}}{\nu_{e e}}$. Their linearized Fokker-Planck collision operator has a pitchangle term and the drag term but no diffusion term.

In the present paper, similar assumptions as Hsu et al. [7] are used except that a more complete form of linearized Coulomb collision operator has been used. The resulting poloidal potential variation is expressed explicitly in terms of temperature anisotropy due to cyclotron resonance heating.

The consequence of this poloidal potential variation can appear in various ways. The most immediate consequence is a significant enhancement in the neoclassical transport coefficients [10-16]. It has also been reported that the poloidal potential variation may affect the plasma stability [17].

For the sake of simplicity, we confine the present analysis to the cases where the inverse aspect ratio, $\epsilon=r / R_{0}$, is small, equilibrium magnetic flux surfaces have a circular cross-section, and background particles (electrons, ions, and single species impurity ions) have the same temperature. Furthermore we assume a zero parallel wave number so that we neglect the Doppler shift effect in the wave-particle interaction.

In Section 2, the anisotropic perpendicular and parallel temperatures are calcu- 
lated using a bi-Maxwellian model of resonant particle distribution. The electric field along a magnetic field line as a function of temperature anisotropy is derived in Section 3. In Section 4, a summary is presented. 


\section{KINETIC CALCULATION OF TEMPERATURE AN- ISOTROPY}

In order to estimate the temperature anisotropy driven by cyclotron resonance heatings, a simple model is considered in this paper. We assume that the background particles obey the Maxwellian distribution, and that the density of the warm particles is small compared to those of the background particles. We will neglect Coulomb self-collisions between the warm species when compared to the collisions of the warm particles against background species. Plasma particles are divided into four different species, background cold electrons $(e)$ and ions $(i)$, cold impurity species $(I)$, and warm electrons or ions $(w)$ depending on the type of input waves.

The $v_{\perp}^{2}$ and $v_{\|}^{2}$ moments of warm species Fokker-Planck equation yield

$$
\begin{array}{r}
\nabla \cdot \mathbf{q}_{\perp}=\frac{1}{2} \int d^{3} v m v_{\perp}^{2}\left(\frac{\partial f}{\partial t}\right), \\
\nabla \cdot \mathbf{q}_{\|}=\frac{1}{2} \int d^{3} v m v_{\|}^{2}\left(\frac{\partial f}{\partial t}\right), \\
\frac{\partial f}{\partial t}=C(f)+Q(f),
\end{array}
$$

where $C$ and $Q$ represent Coulomb collision operator and the quasilinear scattering operator, respectively, and the equilibrium mass flow of warm species has been assumed to be small.

The Coulomb collision operator for a warm particle is expressed as [18]

$$
C(f)=C_{\xi}(f)+C_{\epsilon}(f)
$$

where

$$
\begin{array}{r}
C_{\xi}(f)=\frac{1}{2} \nu_{\xi}^{w / b} \frac{\partial}{\partial \xi}\left(1-\xi^{2}\right) \frac{\partial}{\partial \xi} f \\
C_{\epsilon}(f)=\frac{1}{v^{2}} \frac{\partial}{\partial v} v^{2} \nu_{c}^{w / b}\left(v f+\frac{k T_{b}}{m_{w}} \frac{\partial f}{\partial v}\right) \\
\nu_{\xi}^{w / b}=\nu_{\xi 0}\left(\frac{v_{b}}{v}\right)^{3} \phi_{1}\left(\frac{v}{v_{b}}\right)
\end{array}
$$




$$
\begin{array}{r}
\nu_{\epsilon}^{w / b}=\nu_{\epsilon 0}\left(\frac{v_{b}}{v}\right)^{3} \phi_{2}\left(\frac{v}{v_{b}}\right), \\
\nu_{\xi 0}=\frac{3 \sqrt{\pi}}{4}\left(\frac{m_{b}}{m_{w}}\right)^{2} \frac{1}{\tau_{c, b}}, \\
\nu_{\epsilon 0}=\frac{3 \sqrt{\pi}}{4} \frac{m_{b}}{m_{w}} \frac{1}{\tau_{c, b}}, \\
\phi_{1}(u)=\left(1-\frac{1}{2 u^{2}}\right) \operatorname{Erf}(u)+\frac{e^{-u^{2}}}{\sqrt{\pi} u}, \\
\phi_{2}(u)=\operatorname{Erf}(u)-\frac{2}{\sqrt{\pi}} u e^{-u^{2}}, \\
\frac{1}{\tau_{c, b}}=\frac{4 \sqrt{2 \pi} n_{b} Z_{b}^{2} Z_{w}^{2} e^{4} \ln \Lambda}{3 \sqrt{m_{b}}\left(k T_{b}\right)^{3 / 2}} .
\end{array}
$$

Here, $w$ and $b$ denote warm and background particles $(b=e, i, I) ; C_{\xi}$ and $C_{\epsilon}$ are pitch angle and energy scattering (drag + diffusion) operators; $v_{b}$ is the thermal velocity of the background particles: $T_{b}$ is the background particle temperature; $Z_{w}$ and $Z_{b}$ are the charge number of warm and background particles; $m_{w}$ and $m_{b}$ are mass of warm and background particles respectively; $\xi=v_{\|} / v$; and $\tau_{c, b}$ is the Coulomb collision time at background temperature. The functions, $\phi_{1}(u)$ and $\phi_{2}(u)$ become unity when $u \gg 1$, and their asymptotics for $u \ll 1$ are

$$
\begin{aligned}
& \phi_{1}(u) \approx \frac{4}{3 \sqrt{\pi}} u, \\
& \phi_{2}(u) \approx \frac{4}{3 \sqrt{\pi}} u^{3} .
\end{aligned}
$$

For the quasilinear operator $Q(f)$, we use the homogeneous magnetic field version of Kennel and Engelmann [19].

$$
\begin{array}{r}
Q(f)=\frac{1}{v_{\perp}} \frac{\partial}{\partial v_{\perp}} v_{\perp} D \frac{\partial f}{\partial v_{\perp}} \\
D=\sum_{l} \frac{\pi Z_{w}^{2} e^{2}}{\delta m_{w}^{2}}\left|E_{ \pm}\right|^{2} J_{l \pm 1}^{2}\left(k_{\perp} \rho\right) \delta\left(\omega-k_{\|} v_{\|}-l \Omega\right),
\end{array}
$$

where $E_{+}\left(E_{-}\right)$represents left-handed (right-handed) circularly polarized electric field, $\omega$ is the wave frequency, $D$ the quasilinear velocity-space particle diffusion coefficient driven by cyclotron waves a.t the resonant location, $J_{l \pm 1}$ the Bessel function of the first 
kind of order $l \pm 1, k_{\perp}$ the wave vector perpendicular to the equilibrium magnetic field, and $\rho$ is the gyroradius. The delta function in $D$ requires that the particles should be resonant somewhere along its orbits to have a nonvanishing $D$, and its relation to the absorbed wave power will be shown later in this paper. In Eq. (12), we will assume that the Doppler shift effect is small, $k_{\|} v_{\|}<\omega$.

If we define $\lambda$ as

$$
\lambda \equiv \frac{B_{m}}{B} \frac{v_{\perp}^{2}}{v^{2}}
$$

then $\lambda$ is a constant of motion. We use $(v, \lambda)$ coordinates for particle velocity. Here, $B_{m}$ is the magnetic field at the minimum Mod-B point (outside midplane). The Jacobian in these velocity coordinates is calculated to be

$$
\int d^{3} v=\sum_{\sigma} \int \frac{\pi v^{3}}{\left|v_{\|}\right|} \frac{B}{B_{m}} d \lambda d v
$$

where $\sigma=v_{\|} /\left|v_{\|}\right|$and the range of the integral is $[0, \infty]$ for $v$ and $[0,1]$ for $\lambda$.

In the flux coordinates, the flux surface average of a physical quantity $g$ can be written as

$$
\langle g\rangle=\frac{\int d \theta \mathcal{J} g}{\int d \theta \mathcal{J}}
$$

and the bounce average is defined as

$$
\begin{array}{r}
\{g\}=\frac{1}{\tau_{b}} \int \frac{\mathcal{J} B}{\left|v_{\|}\right|} d \theta g, \\
\tau_{b}=\int \frac{\mathcal{J} B d \theta}{\left|v_{\|}\right|} .
\end{array}
$$

The bounce integral is taken between $-\pi \leq \theta \leq \pi$ for passing particles and $-\theta_{t} \leq \theta \leq$ $\theta_{t}$ for trapped particles where $\theta_{t}$ is the poloidal angle of banana tips. The Jacobian for the flux coordinates $(\psi, \theta, \zeta)$ is expressed as

$$
\mathcal{J}=(\nabla \psi \times \nabla \theta \cdot \nabla \zeta)^{-1}
$$


By changing the order of integration and using the definitions for flux surface average and bounce average, flux surface average over a velocity integration of a quantity $g$ in a strong $B$ field can be reduced to a velocity integral if the bounce-averaged quantity of $g$ is known [20]:

$$
\left\langle\int d^{3} v g\right\rangle=\frac{\pi}{B_{m} \int \mathcal{J} d \theta} \sum_{\sigma} \int d \lambda d v v^{3} \tau_{b}\{g\}
$$

Furthermore, since the integrand for bounce time is proportional to $1 / v_{\|}, \hat{\tau}_{b} \equiv v \tau_{b}$ is independent of $v$, but dependent on $\lambda$.

For the estimation of the anisotropic temperatures, a bi-Maxwellian distribution function (which is constant along field lines) is used for the warm particles.

$$
f=f_{N}\left(\alpha_{\perp}^{2}, \alpha_{\|}^{2}\right) \cdot e^{-v^{2}\left(\frac{\lambda}{\alpha_{\perp}^{2}}+\frac{1-\lambda}{\alpha_{\|}^{2}}\right)}=f_{N} e^{-b(c-\lambda) \frac{v^{2}}{v_{b}^{2}}}
$$

where

$$
b=\frac{s-1}{\alpha_{\perp}^{2} / v_{b}^{2}}, \quad c=\frac{s}{s-1}, \quad s=\frac{\alpha_{\perp}^{2}}{\alpha_{\|}^{2}}=\frac{T_{\perp}}{T_{\|}} .
$$

Here $k T_{\perp}\left(=m_{w} \alpha_{\perp}^{2} / 2\right)$ and $k T_{\|}\left(=m_{w} \alpha_{\|}^{2} / 2\right)$ are effective temperatures perpendicular and parallel to magnetic field lines and $f_{N}$ is the normalization factor. With this form of the distribution function, $\alpha_{\perp}^{2}, \alpha_{\|}^{2}$ can be determined from the perpendicular and parallel energy moment Eqs. (1) and (2),

$$
\begin{aligned}
\left\langle\int d^{3} v v_{\perp}^{2}\left[C_{\xi}+C_{\epsilon}+Q\right](f)\right\rangle & =0, \\
\left\langle\int d^{3} v v_{\|}^{2}\left[C_{\xi}+C_{\epsilon}\right](f)\right\rangle & =0 .
\end{aligned}
$$

Here it is assumed that the $\langle\nabla \cdot q\rangle$ term, which represents the radial heat transport, is small. Most of the energy deposited by wave heating is transferred to background species by collisions.

Since we assume a small Doppler shift effect in $Q$, the particle heating is in the perpendicular direction only and, thus, there is no direct resonance wave heating 
term in the parallel equation [19]. The above two equations (17) and (18) show the appropriate path of energy flow from the input wave to the resonant particles. The $v_{\perp}^{2} Q(f)$ term in Eq. (17) represents the wave heating term and it is positive definite. The Coulomb energy scattering terms then represent energy transfer to the background species and redistribution within the warm species. The pitch angle scattering operator pictures the change of the direction of the particle velocity on an equi-energy surface. Since $v_{\perp}^{2}$ and $v_{\|}^{2}$ are multiplied to the operator, the terms $v_{\perp}^{2} C_{\xi}(f), v_{\|}^{2} C_{\xi}(f)$ should show transfer of perpendicular energy which is increased by quasilinear wave heating to the parallel direction. This means the two terms have the same magnitudes but opposite signs.

Using Eq. (15), Eqs. (17) and (18) can be expressed as

$$
\begin{array}{r}
\int_{0}^{1} d \lambda \int_{0}^{\infty} d v v^{2} v \tau_{b}\left[\left\{v_{\perp}^{2} C_{\xi}\right\}+\left\{v_{\perp}^{2} C_{\epsilon}\right\}\right](f)+H_{\perp}=0 \\
\int_{0}^{1} d \lambda \int_{0}^{\infty} d v v^{2} v \tau_{b}\left[\left\{v_{\|}^{2} C_{\xi}\right\}+\left\{v_{\|}^{2} C_{\epsilon}\right\}\right](f)=0
\end{array}
$$

where

$$
H_{\perp}=\left(\frac{\pi}{B_{m} \int \mathcal{J} d \theta}\right)^{-1}\left\langle\int d^{3} v v_{\perp}^{2} Q(f)\right\rangle
$$

The bounce time (transit time for passing particles) is calculated to be

$$
\hat{\tau}_{b} \equiv v \tau_{b}= \begin{cases}\frac{2}{\pi} \sqrt{1+2 x} K(x) & (x<1) \\ \frac{2}{\pi} \sqrt{\frac{1+2 x}{x}} K\left(\frac{1}{x}\right) & (x>1),\end{cases}
$$

where $K(x)$ is the complete elliptic integral of the first kind with modulus $x$, and the variable $x$ is defined as

$$
x=\frac{2 \epsilon}{1-\epsilon} \frac{\lambda}{1-\lambda} .
$$

$x=1$ describes the boundary between trapped and passing particles, with $x>1$ describing the trapped particles. For numerical evaluations in this work, we choose $\epsilon=1 / 5$. 
As can seen in Fig. 1(a), $\hat{\tau}_{b}$ has a singularity at the trapped-passing boundary. In order to avoid numerical problems associated with this singularity, we replace $\hat{\tau}_{b}$ with a well behaved function $V_{t t}(\lambda)$. The smoothed function, $V_{t t}(\lambda)$ is obtained by fitting $\hat{\tau}_{b}$ based on polynomials of $\lambda$ and is plotted with dotted line.

Notice here that the operation $C_{\epsilon}(f)$ can be taken out of the bounce integral since it is only a function of $v$ and, thus, constant of motion,

$$
\begin{aligned}
& \left\{v_{\perp}^{2} C_{\epsilon}\right\}=\left\{v_{\perp}^{2}\right\} C_{\epsilon}, \\
& \left\{v_{\|}^{2} C_{\epsilon}\right\}=\left\{v_{\|}^{2}\right\} C_{\epsilon} .
\end{aligned}
$$

The functions $\left\{v_{\perp}^{2}\right\}$ and $\left\{v_{\|}^{2}\right\}$ are calculated and approximated again in order to avoid singularities:

$$
\begin{aligned}
& \frac{\hat{\tau}_{b}}{v^{2}}\left\{v_{\|}^{2}\right\}=\left\{\begin{array}{cl}
\frac{2}{\pi} \frac{1}{\sqrt{1+2 x}} E(x) & (x<1), \\
\frac{2}{\pi} \sqrt{\frac{x}{1+2 x}}\left[E\left(\frac{1}{x}\right)-\left(1-\frac{1}{x}\right) K\left(\frac{1}{x}\right)\right] & (x>1),
\end{array}\right. \\
& \approx V_{t l}(\lambda) \text {, } \\
& \frac{\hat{r_{b}}}{v^{2}}\left\{v_{\perp}^{2}\right\}=\left\{\begin{array}{cl}
\frac{2}{\pi} \sqrt{1+2 x} K(x)-\frac{2}{\pi} \frac{1}{\sqrt{1+2 x}} E(x) & (x<1), \\
\frac{2}{\pi} \sqrt{\frac{1+2 x}{x}} K\left(\frac{1}{x}\right)-\frac{2}{\pi} \sqrt{\frac{x}{1+2 x}}\left[E\left(\frac{1}{x}\right)-\left(1-\frac{1}{x}\right) K\left(\frac{1}{x}\right)\right] & (x>1),
\end{array}\right. \\
& \approx V_{t p}(\lambda),
\end{aligned}
$$

where $E(x)$ is the complete elliptic integral of the second kind.

By the chain rule, the pitch angle scattering operator, $E q$. (3), can be expressed in terms of the new coordinates, $(v, \lambda)$

$$
C_{\xi}=2 \nu_{\xi} \frac{B_{m}}{B} \xi \frac{\partial}{\partial \lambda} \lambda \xi \frac{\partial}{\partial \lambda},
$$

where $\xi=\sigma \sqrt{1-\frac{B}{B_{m}} \lambda}$ and the $90^{\circ}$ Coulomb collision frequency $\nu_{\xi}$ is a function of $v$ only. 
Using the relations,

$$
\frac{v_{\|}^{2}}{v^{2}}=\xi^{2}=1-\frac{B}{B_{m}} \lambda, \quad \frac{v_{\perp}^{2}}{v^{2}}=1-\xi^{2}=\frac{B}{B_{m}} \lambda
$$

we obtain bounce average of $v_{\perp}^{2} C_{\xi}(f)$ and $v_{\|}^{2} C_{\xi}(f)$ as

$$
\begin{array}{r}
\hat{\tau}_{b}\left\{v_{\perp}^{2} C_{\xi}(f)\right\}=2 \nu_{\xi} v^{2} \lambda\left[-\hat{\tau}_{b} \frac{1}{2} \frac{\partial f}{\partial \lambda}+\frac{\hat{\tau_{b}}}{v^{2}}\left\{v_{\|}^{2}\right\}\left(\frac{\partial}{\partial \lambda} \lambda \frac{\partial f}{\partial \lambda}+\frac{1}{2} \frac{\partial f}{\partial \lambda}\right)\right], \\
\hat{\tau}_{b}\left\{v_{\|}^{2} C_{\xi}(f)\right\}=2 \nu_{\xi} v^{2}\left[\frac{\hat{\tau}_{b}}{v^{2}}\left\{\frac{B_{m}}{B} v_{\|}^{2}\right\} \frac{\partial}{\partial \lambda} \lambda \frac{\partial f}{\partial \lambda}-\frac{\hat{\tau}_{b}}{v^{2}}\left\{v_{\|}^{2}\right\} \lambda\left(\frac{\partial}{\partial \lambda} \lambda \frac{\partial f}{\partial \lambda}+\frac{1}{2} \frac{\partial f}{\partial \lambda}\right)\right] .
\end{array}
$$

By a similar procedure, $\frac{\hat{\tau}_{b}}{v^{2}}\left\{\frac{B_{m}}{B} v_{\|}^{2}\right\}$ can be obtained, using an $\epsilon$-expansion of $\frac{B_{m}}{B}$ up to the first order in $\epsilon$,

$$
\begin{aligned}
& \frac{\hat{\tau}_{b}}{v^{2}}\left\{\frac{B_{m}}{B} v_{\|}^{2}\right\}=\left\{\begin{array}{cc}
\frac{2}{\pi} \frac{1}{\sqrt{1+2 x}}\left[E(x)-\frac{\pi}{10}{ }_{2} F_{1}\left(-\frac{1}{2}, \frac{3}{2}, 2, x\right)\right] & (x<1), \\
\frac{2}{\pi} \sqrt{\frac{x}{1+2 x}}\left[E\left(\frac{1}{x}\right)-\left(1-\frac{1}{x}\right) K\left(\frac{1}{x}\right)-\frac{\pi}{40} \frac{1}{x^{2}}{ }_{2} F_{1}\left(\frac{1}{2}, \frac{3}{2}, 3, \frac{1}{x}\right)\right] & (x>1),
\end{array}\right. \\
& \approx V_{t b}(\lambda)
\end{aligned}
$$

where $F$ is the hypergeometric function. Since the bounce time, $\hat{\tau}_{b}$ diverges at the trapped-passing boundary, $\left\{v_{\|}^{2}\right\}$ and $\left\{\frac{B_{m}}{B} v_{\|}^{2}\right\}$ approach 0 as expected.

The bounce-averaged quantities, Eqs. (22), (25), (26), and (29), are depicted in Fig. 1 with solid lines. Their fitted functions of the form with constants $c_{0}, c_{1}, c_{2}$, and $c_{3}$,

$$
V_{t t}, V_{t l}, V_{t p}, V_{t b}=c_{0}+c_{1} \lambda+c_{2} \lambda^{2}+c_{3} \lambda^{3}
$$

are also plotted with dotted lines.

In the present model $\left(k_{\|} v_{\|} \simeq 0\right)$, perpendicular heatings occur at the resonance layer. Due to the conservation of the magnetic moment along the guiding center motion in a toroidal system, however, the perpendicular heating at the resonance location appears differently along the magnetic field line, resulting in a varying degree of the temperature anisotropy along the magnetic field line. In the present work we calculate the amount of anisotropy at the resonance point for the sake of simplicity. 
In order to manipulate the delta function in $D$, we expand [21] the resonance condition $l \Omega-\omega=0$ near the resonance layer whose poloidal angle is given by $\theta_{R}$,

$$
l \Omega-\left.\omega \simeq l \frac{d \Omega}{d \theta}\right|_{\theta=\theta_{R}}\left(\theta-\theta_{R}\right)=G \cdot\left(\theta-\theta_{R}\right),
$$

where the frequency, $G$ incorporated with geometrical factor is,

$$
G=\omega \epsilon \frac{\sin \theta_{R}}{1-\epsilon \cos \theta_{R}}
$$

and $B=\frac{B_{0}}{1+\epsilon \cos \theta}$ is used. If we write $D=D_{0} \delta\left(\omega-k_{\|} v_{\|}-l \Omega\right)$ and assume that the quasilinear diffusion coefficient, $D_{0}\left(=\frac{\pi Z_{w}^{2} e^{2}}{8 m_{w}^{2}}\left|E_{ \pm}\right|^{2}\right.$ assuming $\left.k_{\perp} \rho \ll 1\right)$ is constant [22] with respect to $v$ and $\lambda$, the velocity integral of $v_{\perp}^{2} Q(f)$ becomes

$$
\int d^{3} v v_{\perp}^{2} Q(f)=\left.\frac{4 D_{0}}{G} \int d^{3} v f\right|_{\theta=\theta_{R}} .
$$

Finally, the heating term, Eq. (21), is given as

$$
\left\langle\int d^{3} v v_{\perp}^{2} Q(f)\right\rangle=\left.\frac{4 D_{0}}{G} \frac{\pi}{B_{m} \int \mathcal{J} d \theta} \int d \lambda d v v^{2} \hat{\tau}_{b} f\right|_{\theta=\theta_{R}} .
$$

In summary, the two simultaneous equations (19) and (20) are to be solved in order to obtain the anisotropic temperatures. The necessary bounce-averaged quantities are approximated in the form of Eq. (30). The heating term is derived in $E q$. (21) and Eq. (31).

Depending on the type of resonant particles, i.e., electrons with ECRH and ions with ICRH, different approximations apply to the Coulomb collision operators, so we consider ion and electron cyclotron wave heatings separately.

\subsection{Ion Cyclotron Resonance Heating}

For ICRH, the pitch angle scattering of the warm ions is by collisions between warm ions and background ions (including single species impurity ions) rather than 
with cold electrons, i.e.,

$$
\frac{\nu_{\xi}^{w / e}}{\nu_{\xi}^{w / i}}=\frac{n_{e}}{n_{i} Z_{i}^{2}} \frac{\phi_{1}\left(\frac{v}{v_{e}} \ll 1\right)}{\phi_{1}\left(\frac{v}{v_{i}} \geq 1\right)} \ll 1,
$$

and $\phi_{1}(u)$ is in the range of $0.6 \leq \phi_{1}(u) \leq 1$ for $u=\frac{v}{v_{i}} \geq 1$, where $v_{i}$ is the background ion thermal velocity. We will assume $T_{i} \approx T_{e} \approx T_{I}$ for simplicity. The characteristic frequency for pitch angle scattering is, thus,

$$
\begin{aligned}
\nu_{\xi} \approx \nu_{\xi}^{w / i}+\nu_{\xi}^{w / I} & \approx\left(1+\frac{n_{I} Z_{I}^{2}}{n_{i} Z_{i}^{2}}\right) \nu_{\xi}^{w / i} \\
& =\nu_{\xi 1}\left(\frac{v_{i}}{v}\right)^{3} \phi_{1}\left(\frac{v}{v_{i}}\right),
\end{aligned}
$$

where

$$
\begin{array}{r}
\nu_{\xi 1}=\nu_{0}\left(\frac{m_{i}}{m_{w}}\right)^{2}\left[1+\frac{n_{I} Z_{I}^{2}}{n_{i} Z_{i}^{2}}\right], \quad \nu_{0}=\frac{3 \sqrt{\pi}}{4} \frac{1}{\tau_{i}} \\
\frac{1}{\tau_{i}}=\frac{4 \sqrt{2 \pi} n_{i} Z_{i}^{2} Z_{w}^{2} e^{4} \ln \Lambda}{3 \sqrt{m_{i}}\left(k T_{i}\right)^{3 / 2}} .
\end{array}
$$

In the energy operator, however, collisions with background electrons cannot be neglected, and the Coulomb energy operator can be shown to be

$$
C_{\epsilon}(f)=\nu_{\epsilon 1} \frac{1}{u^{2}} \frac{\partial}{\partial u}\left[\phi_{2}(u)\left(f+\frac{k T_{i}}{m_{i}} \frac{1}{v_{i}^{2}} \frac{1}{u} \frac{\partial f}{\partial u}\right)+\left(\frac{m_{i}}{m_{e}}\right) \frac{1}{Z_{e}} \phi_{2}\left(\frac{v_{i}}{v_{e}} u\right)\left(f+\frac{k T_{e}}{m_{i}} \frac{1}{v_{i}^{2}} \frac{1}{u} \frac{\partial f}{\partial u}\right)\right],
$$

where

$$
\begin{array}{r}
\nu_{c 1}=\nu_{0}\left(\frac{m_{i}}{m_{w}}\right)\left[1+\left(\frac{m_{i}}{m_{I}}\right)\left(\frac{n_{I} Z_{I}^{2}}{n_{i} Z_{i}^{2}}\right)\right], \quad \frac{1}{Z_{e}}=\left(\frac{n_{e}}{n_{i} Z_{i}^{2}}\right) /\left[1+\left(\frac{m_{i}}{m_{I}}\right)\left(\frac{n_{I} Z_{I}^{2}}{n_{i} Z_{i}^{2}}\right)\right] \\
u=\frac{v}{v_{i}} .
\end{array}
$$

Then, from $E q s .(24)$ - (2S)

$$
\begin{array}{r}
\hat{\tau}_{b}\left\{v_{\perp}^{2} C_{\xi}(f)\right\}=\frac{2 \nu_{\xi 1} v_{i}^{2}}{u} \phi_{1}(u) \lambda\left[-V_{t t}(\lambda) \frac{1}{2} \frac{\partial f}{\partial \lambda}+V_{t l}(\lambda)\left(\frac{\partial}{\partial \lambda} \lambda \frac{\partial f}{\partial \lambda}+\frac{1}{2} \frac{\partial f}{\partial \lambda}\right)\right] \\
\hat{\tau}_{b}\left\{v_{\|}^{2} C_{\xi}(f)\right\}=\frac{2 \nu_{\xi 1} v_{i}^{2}}{u} \phi_{1}(u)\left[V_{t b}(\lambda) \frac{\partial}{\partial \lambda} \lambda \frac{\partial f}{\partial \lambda}-V_{t l}(\lambda) \lambda\left(\frac{\partial}{\partial \lambda} \lambda \frac{\partial f}{\partial \lambda}+\frac{1}{2} \frac{\partial f}{\partial \lambda}\right)\right] \\
\hat{\tau}_{b}\left\{v_{\perp}^{2} C_{\epsilon}(f)\right\}=v_{i}^{2} u^{2} V_{t p}(\lambda) C_{\epsilon}(f) \\
\hat{\tau}_{b}\left\{v_{\|}^{2} C_{\epsilon}(f)\right\}=v_{i}^{2} u^{2} V_{t l}(\lambda) C_{\epsilon}(f) .
\end{array}
$$


Because of the functions $\phi_{1}(u)$ and $\phi_{2}(u)$, it is not straightforward to evaluate the integrals in Eqs. (19) and (20) by hand. Instead, we use the mathematical software, Mathematica $^{T M}$ by Wolfram Research, Inc. [23] which has the capability of doing symbolic differentiation and integration. Inserting Eqs. (32) - (35) into Eqs. (19) and (20) with the assumed bi-Maxwellian warm particle distribution function, Eq. (16), we get two simultaneous equations for the temperature ratio, $s$, and the perpendicular energy, $m_{w} \alpha_{\perp}^{2} / 2$ :

$$
\begin{array}{r}
-\gamma_{1} T_{p a, i}^{\perp}+\gamma_{2}\left(-T_{d r, i}^{\perp}+T_{d j, i}^{\perp}\right)+\gamma_{3}\left(-T_{d r, e}^{\perp}+T_{d f, e}^{\perp}\right)+H_{\perp}^{i}=0 \\
\gamma_{1} T_{p a, i}^{\|}+\gamma_{2}\left(-T_{d r, i}^{\|}+T_{d j, i}^{\|}\right)+\gamma_{3}\left(-T_{d r, e}^{\|}+T_{d j, e}^{\|}\right)=0
\end{array}
$$

where

$$
\begin{array}{r}
H_{\perp}^{i}=d_{i} \cdot H_{t}\left(s, \frac{\alpha_{\perp}^{2}}{v_{i}^{2}}\right) f_{N}, \\
d_{i}=\frac{4 D_{0}}{G} \frac{1}{\nu_{0} v_{i}^{2}}, \\
H_{t}\left(s, \frac{\alpha_{\perp}^{2}}{v_{i}^{2}}\right)=\int_{0}^{1} d \lambda V_{t t}(\lambda) \int_{0}^{\infty} d u u^{2} e^{-b(c-\lambda) u^{2},} \\
T_{p a, i}^{\perp}=T_{11}-T_{12}, \\
T_{p a, i}^{\|}=T_{21}-T_{22}, \\
T_{d f, i}^{\perp}=2 b \int_{0}^{1} d \lambda V_{t p}(\lambda)(c-\lambda) \int_{0}^{\infty} d u u \phi_{2}(u) f, \\
T_{d r, \epsilon}^{\perp}=2 \int_{0}^{1} d \lambda V_{t p}(\lambda) \int_{0}^{\infty} d u u^{4} f, \\
T_{d j, \epsilon}^{\perp}=3 \int_{0}^{1} d \lambda V_{t p}(\lambda) \int_{0}^{\infty} d u u^{2} f, \\
T_{d r, i}^{\|}=2 \int_{0}^{1} d \lambda V_{t l}(\lambda) \int_{0}^{\infty} d u u \phi_{2}(u) f, \\
T_{d f, i}^{\|}=2 b \int_{0}^{1} d \lambda V_{t l}(\lambda)(c-\lambda) \int_{0}^{\infty} d u u \phi_{2}(u) f, \\
T_{d r, e}^{\|}=2 \int_{0}^{1} d \lambda V_{t l}(\lambda) \int_{0}^{\infty} d u u^{4} f, \\
T_{d f, e}^{\|}=3 \int_{0}^{1} d \lambda V_{t l}(\lambda) \int_{0}^{\infty} d u u^{2} f,
\end{array}
$$




$$
\begin{array}{r}
T_{11}=\int_{0}^{1} d \lambda \lambda V_{t t}(\lambda) \int_{0}^{\infty} d u u \phi_{1}(u) \frac{\partial f}{\partial \lambda} \\
T_{12}=2 \int_{0}^{1} d \lambda \lambda V_{t l}(\lambda) \int_{0}^{\infty} d u u \phi_{1}(u)\left(\frac{\partial}{\partial \lambda} \lambda \frac{\partial f}{\partial \lambda}+\frac{1}{2} \frac{\partial f}{\partial \lambda}\right) \\
T_{21}=2 \int_{0}^{1} d \lambda V_{t b}(\lambda) \int_{0}^{\infty} d u u \phi_{1}(u) \frac{\partial}{\partial \lambda} \lambda \frac{\partial f}{\partial \lambda} \\
\cdot \quad T_{22}=T_{12} \\
b=\frac{s-1}{\alpha_{\perp}^{2} / v_{i}^{2}}, \quad c=\frac{s}{s-1}, \\
\gamma_{1}=\left(\frac{m_{i}}{m_{w}}\right)^{2}\left[1+\left(\frac{n_{I} Z_{I}^{2}}{n_{i} Z_{i}^{2}}\right)\right], \\
\gamma_{2}=\left(\frac{m_{i}}{m_{w}}\right)\left[1+\left(\frac{m_{i}}{m_{I}}\right)\left(\frac{n_{I} Z_{I}^{2}}{n_{i} Z_{i}^{2}}\right)\right], \\
\gamma_{3}=\frac{4}{3 \sqrt{\pi}}\left(\frac{m_{e}}{m_{i}}\right)^{1 / 2}\left(\frac{m_{i}}{m_{w}}\right)\left(\frac{n_{e}}{n_{i} Z_{i}^{2}}\right) .
\end{array}
$$

The first subscripts, $p a, d r$, and $d f$ indicate pitch angle, drag, and diffusion, respectively, and the second subscript after a comma denotes the species of background particles with which warm ions collide. One can notice that $E q$. (41) - Eq. (50) are all positive definite since $b, c \geq 1$. The density ratios in $\gamma_{1}, \gamma_{2}, \gamma_{3}$ can be expressed in terms of $Z_{\text {eff }}, Z_{i}, Z_{I}$ by the definition of $Z_{\text {eff }}$ and the quasineutrality condition,

$$
\begin{aligned}
& \frac{n_{I} Z_{I}^{2}}{n_{i} Z_{i}^{2}}=\frac{Z_{I}}{Z_{i}} \frac{Z_{e f f}-Z_{i}}{Z_{I}-Z_{e f f}} \\
& \frac{n_{e}}{n_{i} Z_{i}^{2}}=\frac{1}{Z_{i}} \frac{Z_{I}-Z_{i}}{Z_{I}-Z_{e f f}} .
\end{aligned}
$$

From Eqs. (36) and (37), one can see that the pitch angle term becomes bigger with a larger fraction of impurity species since the coefficient $\gamma_{1}$ increases with $Z_{\text {eff }}$, which results in less anisotropy in temperatures. In addition, $\gamma_{2}, \gamma_{3}$ are also increasing functions of $Z_{\text {eff }}$ and it turns out that $\left(-T_{d r}^{\perp}+T_{d f}^{\perp}\right)<\left(-T_{d r}^{\|}+T_{d f}^{\|}\right)<0$. It means that the wave heating can become less effective with larger $Z_{\text {eff }}$ since Coulomb energy scattering makes warm ion energy flow to the background more easily.

If we consider that the warm particle density is given by

$$
n_{w}=\langle n(\theta)\rangle=\left\langle\int d^{3} v f\right\rangle
$$


the absorbed wave power can be derived by $E q .(31)$,

$$
\left\langle P_{\perp}\right\rangle=\frac{1}{2} m_{w}\left\langle\int d^{3} v v_{\perp}^{2} Q(f)\right\rangle=\frac{m_{w}}{2} \frac{4 D_{0}}{G} n_{w}
$$

Therefore, the parameter, $d_{i}$ which is a function of the wave power and warm particle density is expressed as

$$
d_{i}=\frac{\left\langle P_{\perp}\right\rangle}{n_{w} k T_{i} \nu_{0}}\left(\frac{m_{i}}{m_{w}}\right)
$$

Figure 2 to Figure 5 are obtained by solving $E q .(36)$ and $E q$. (37) in the case of main ion heating, i.e. $m_{w^{\prime}}=m_{i}$. Figures 2 and 3 depict the perpendicular and parallel temperatures and their ratio as a function of $d_{i}$ with various $Z_{\text {eff }}$ values in a hydrogen and a deuterium plasma ( $\epsilon=1 / 5$ is used). For the impurity species, carbon is used. As shown in the figures, beyond a certain value of $d_{i}$, no solution can be obtained, which suggest that there exists a critical wave power below which our bi-Maxwellian picture holds. In order to extend the present analysis above this critical power, selfcollisions between the warm particles and/or a better description of the distribution for warm species may be necessary.

Figures 4 and 5 show the perpendicular and the parallel temperatures, and the temperature ratio of warm ions, respectively. From these figures, one can notice several effects of impurity ion contribution through $Z_{\text {eff }}$ and the ion mass.

First, the greater the impurity level in a plasma (larger $Z_{\text {eff }}$ ), the higher the $T_{\|}$(and lower $T_{\perp}$ ), and thus less anisotropy is obtained. This is because the pitch angle scattering term $\left(\gamma_{1}\right)$ increases with $Z_{\text {eff }}$ more rapidly than the energy scattering terms $\left(\gamma_{1}, \gamma_{2}\right)$ resulting in a more effective spread of warm ion energy to the parallel direction.

Second, as depicted in Fig. $4(b)$, the parallel temperature reaches a maximum value and decreases with $d_{i}$. This is because when the wave power is large, the 
transferred power from the perpendicular to the parallel direction decreases:

$$
P_{\|} \sim \nu_{\xi} k\left(T_{\perp}-T_{\|}\right) \propto \frac{1}{\sqrt{k T_{\perp}}}
$$

where $\nu_{\xi}$ is proportional to $1 / v^{3}$.

Third, since a deuterium ion loses less energy to the background electrons than a hydrogen ion due to the heavier mass, one can expect more efficient heating of a deuterium plasma than a hydrogen plasma at the same wave power. Furthermore, the relation $\nu_{\xi} \propto 1 / \sqrt{m_{w}}$ gives higher temperature anisotropy for deuterium ions as seen in Fig. 5 at a fixed $d_{i}$.

Finally, the parameter $d_{i}$ is

$$
d_{i} \propto \frac{\left\langle P_{\perp}\right\rangle}{n_{w}} \frac{\sqrt{m_{i} k T_{i}}}{n_{i}}
$$

so that higher anisotropy can be expected with higher background temperature, heavier ion mass, or smaller background density.

\subsection{Electron Cyclotron Resonance Heating}

When the resonant particles are electrons, the pitch angle scattering of the warm electrons by background electrons is comparable to that by cold ions unless $Z_{\text {eff }}$ is very large:

$$
\frac{\nu_{\xi}^{w / e}}{\nu_{\xi}^{w / i}+\nu_{\xi}^{u / I}} \approx \frac{1}{Z_{e f f}} \phi_{1}\left(\frac{v}{v_{e}}\right) \sim \frac{1}{Z_{e f f}},
$$

where $v_{e}$ is the background electron thermal velocity. Thus, the two contributions should be equally considered in pitch angle scattering. For the energy scattering operator, the contribution from the background electrons is dominant over that from the cold ions by the ion-electron mass ratio,

$$
\frac{\nu_{\epsilon}^{w / e}}{\nu_{\epsilon}^{w / i}} \approx \frac{1}{Z_{e f f}}\left(\frac{m_{i}}{m_{\epsilon}}\right) \phi_{2}\left(\frac{v}{v_{e}}\right) \gg 1
$$


Thus, the ion term can be ignored in the warm electron energy scattering operator.

Therefore, the pitch angle scattering and the energy scattering characteristic frequencies can be simplified to be

$$
\begin{array}{r}
\nu_{\xi} \approx \nu_{0} \frac{1}{u^{3}}\left(Z_{e f f}+\phi_{1}(u)\right), \\
\nu_{\epsilon} \approx \nu_{0} \frac{1}{u^{3}} \phi_{2}(u), \\
u=\frac{v}{v_{\epsilon}},
\end{array}
$$

where

$$
\nu_{0}=\frac{3 \sqrt{\pi}}{4} \frac{1}{\tau_{e}}, \quad \frac{1}{\tau_{e}}=\frac{4 \sqrt{2 \pi} n_{e} e^{4} \ln \Lambda}{3 \sqrt{m_{e}}\left(k T_{e}\right)^{3 / 2}} .
$$

The first term in $\nu_{\xi}$ (which has $Z_{e f f}$ ) is from the scattering between warm electrons and background ions, and the second term is from that with background electrons.

Then, Eqs. (24) - (28) become

$$
\begin{array}{r}
\hat{\tau}_{b}\left\{v_{\perp}^{2} C_{\xi}(f)\right\}=\frac{2 \nu_{0} v_{e}^{2}}{u}\left(Z_{e f f}+\phi_{1}(u)\right) \lambda\left[-V_{t t}(\lambda) \frac{1}{2} \frac{\partial f}{\partial \lambda}+V_{t l}(\lambda)\left(\frac{\partial}{\partial \lambda} \lambda \frac{\partial f}{\partial \lambda}+\frac{1}{2} \frac{\partial f}{\partial \lambda}\right)\right], \\
\hat{\tau}_{b}\left\{v_{\|}^{2} C_{\xi}(f)\right\}=\frac{2 \nu_{0} v_{e}^{2}}{u}\left(Z_{e f f}+\phi_{1}(u)\right)\left[V_{t b}(\lambda) \frac{\partial}{\partial \lambda} \lambda \frac{\partial f}{\partial \lambda}-V_{t l}(\lambda) \lambda\left(\frac{\partial}{\partial \lambda} \lambda \frac{\partial f}{\partial \lambda}+\frac{1}{2} \frac{\partial f}{\partial \lambda}\right)\right], \\
\tau_{b}\left\{v_{\perp}^{2} C_{\epsilon}(f)\right\}=\nu_{0} v_{e}^{2} V_{t p}(\lambda) \frac{\partial}{\partial u} \phi_{2}(u)\left(f+\frac{1}{2 u} \frac{\partial f}{\partial u}\right), \\
\dot{\tau}_{b}\left\{v_{\|}^{2} C_{\epsilon}(f)\right\}=\nu_{0} v_{e}^{2} V_{t l}(\lambda) \frac{\partial}{\partial u} \phi_{2}(u)\left(f+\frac{1}{2 u} \frac{\partial f}{\partial u}\right) .
\end{array}
$$

As for the ion case, inserting Eqs. (56) - (59) into Eqs. (19) and (20) will give two simultaneous equations to solve,

$$
\begin{array}{r}
-\left(T_{p a, e}^{\perp}+Z_{e f f} T_{p a, i}^{\perp}\right)+\left(-T_{d r, e}^{\perp}+T_{d f, e}^{\perp}\right)+H_{\perp}^{e}=0, \\
\left(T_{p a, e}^{\|}+Z_{e f f} T_{p a, i}^{\|}\right)+\left(-T_{d r, e}^{\|}+T_{d j, e}^{\|}\right)=0,
\end{array}
$$

where

$$
H_{\perp}^{e}=d_{e} \cdot H_{t}\left(s, \frac{\alpha_{\perp}^{2}}{v_{e}^{2}}\right) f_{N}
$$




$$
\begin{aligned}
& d_{e}=\frac{4 D_{0}}{G} \frac{1}{\nu_{0} v_{e}^{2}}=\frac{\left\langle P_{\perp}\right\rangle}{n_{w} k T_{e} \nu_{0}}, \\
& T_{p a, e}^{\perp}=T_{11}-T_{12} \\
& T_{p a, i}^{\perp}=T_{13}-T_{14} \text {, } \\
& T_{p a, e}^{\|}=T_{21}-T_{22}, \\
& T_{p a, i}^{\|}=T_{23}-T_{24}, \\
& T_{d r, e}^{\perp}=2 \int_{0}^{1} d \lambda V_{t p}(\lambda) \int_{0}^{\infty} d u u \phi_{2}(u) \dot{f}, \\
& T_{d f, e}^{1}=2 b \int_{0}^{1} d \lambda V_{t p}(\lambda)(c-\lambda) \int_{0}^{\infty} d u u \phi_{2}(u) f, \\
& T_{d r, e}^{\| l}=2 \int_{0}^{1} d \lambda V_{t l}(\lambda) \int_{0}^{\infty} d u u \phi_{2}(u) f, \\
& T_{d j, e}^{\|}=2 b \int_{0}^{1} d \lambda V_{t l}(\lambda)(c-\lambda) \int_{0}^{\infty} d u u \phi_{2}(u) f, \\
& T_{11}=\int_{0}^{1} d \lambda \lambda V_{t t}(\lambda) \int_{0}^{\infty} d u u \phi_{1}(u) \frac{\partial f}{\partial \lambda}, \\
& T_{12}=2 \int_{0}^{1} d \lambda \lambda V_{t l}(\lambda) \int_{0}^{\infty} d u u \phi_{1}(u)\left(\frac{\partial}{\partial \lambda} \lambda \frac{\partial f}{\partial \lambda}+\frac{1}{2} \frac{\partial f}{\partial \lambda}\right) \text {, } \\
& T_{13}=\int_{0}^{1} d \lambda \lambda V_{t t}(\lambda) \int_{0}^{\infty} d u u \frac{\partial f}{\partial \lambda}, \\
& T_{14}=2 \int_{0}^{1} d \lambda \lambda V_{t l}(\lambda) \int_{0}^{\infty} d u u\left(\frac{\partial}{\partial \lambda} \lambda \frac{\partial f}{\partial \lambda}+\frac{1}{2} \frac{\partial f}{\partial \lambda}\right) \text {, } \\
& T_{21}=2 \int_{0}^{1} d \lambda \lambda V_{t b}(\lambda) \int_{0}^{\infty} d u u \phi_{1}(u) \frac{\partial}{\partial \lambda} \lambda \frac{\partial f}{\partial \lambda}, \\
& T_{22}=T_{12} \text {, } \\
& T_{23}=2 \int_{0}^{1} d \lambda \lambda V_{t b}(\lambda) \int_{0}^{\infty} d u u \frac{\partial}{\partial \lambda} \lambda \frac{\partial f}{\partial \lambda}, \\
& T_{24}=T_{14} \text {, } \\
& b=\frac{s-1}{\alpha_{\perp}^{2} / v_{e}^{2}}, \quad c=\frac{s}{s-1} \text {. }
\end{aligned}
$$

Shown in Fig. 6 is the solution of Eqs. (60) and (61). One important observation to point out here is that the warm electron temperatures run-away at $d_{e} \simeq 0.61$. This run-away effect is expected be originated from the fact that the warm particle density, $n_{w}$, is kept constant with respect to the wave power. We should remember here that the electron energy scattering rate decreases with increasing energy $\left(\nu_{\epsilon} \propto v^{-3}\right)$. 
However, we used a Maxwellian assumption for the resonant species with no cooling mechanisms other than collisions with the background electrons. Hence, for wave power above the run-away power, the validity of the Maxwellian assumption is in doubt.

In the case when there is a strong loss mechanism for the tail electrons, an excessively strong tail formation can be avoided and the Maxwellian treatment may be extended to higher wave power range. 


\section{GENERATION OF EQUILIBRIUM ELECTROSTATIC POTENTIAL PARALLEL TO MAGNETIC FIELD}

In this section, fluid equations are used to evaluate the electrostatic potential variation on a flux surface. Since the warm particles are not in thermal equilibrium, their pressure may be significantly anisotropic.

The steady-state force balance equation for warm particles assuming no plasma flow is written as

$$
\nabla \cdot \mathbf{P}-n_{w} q_{u} \mathrm{E}=0
$$

where $\mathbf{P}=n_{w} k \mathbf{T}$ is the pressure tensor, $q_{w}\left(=Z_{w} e\right)$ the electrical charge of the warm species, $T$ the warm particle temperature, $\mathrm{E}=-\nabla \Phi$, and the collisional friction force is assumed to be weak for the warm resonant particles. Using the assumption that the pressure tensor is diagonal [24],

$$
\mathbf{P}=(\mathbf{I}-\hat{\mathbf{b}} \hat{\mathbf{b}}) p_{\perp}+\hat{\mathbf{b}} \hat{\mathbf{b}} p_{\|},
$$

where $\hat{\mathbf{b}}=\mathbf{B} / B$. The parallel component of $E q .(65)$ in $(\psi, \theta, \zeta)$ coordinates where $\psi, \theta, \zeta$ are radial, poloidal, and toroidal coordinates, respectively, yields

$$
\frac{\partial p_{\|}}{\partial \theta}+\frac{p_{\perp}-p_{\|}}{B} \frac{\partial B}{\partial \theta}=-n_{w} q_{w} \frac{\partial \Phi}{\partial \theta} .
$$

Using the equilibrium magnetic field, with circular cross-sectional flux surfaces, expressed as

$$
B=\frac{B_{0}}{1+\epsilon \cos \theta}
$$

we expand pressure, density, and electrostatic potential in $\epsilon$,

$$
\begin{gathered}
n_{w}=\bar{n}_{w}(\psi)+\tilde{n}_{w}(\theta), \\
p=\bar{p}(\psi)+\tilde{p}(\theta), \\
\Phi=\bar{\Phi}(\psi)+\tilde{\Phi}(\theta),
\end{gathered}
$$


with the ordering

$$
\frac{\tilde{n}_{w}}{\bar{n}_{w}} \sim \frac{\tilde{p}}{\bar{p}} \sim \frac{\tilde{\Phi}}{k T} \sim \epsilon \ll 1
$$

where $\left(\bar{n}_{w}, \bar{p}, \bar{\Phi}\right)$ is the $\theta$-averaged part of $\left(n_{w}, p, \Phi\right)$ and $\left(\tilde{n}_{w}, \tilde{p}, \tilde{\Phi}\right)$ is the $\theta$-varying, first order, part in $\epsilon$. Then, the first order terms of $E q$. (66) yields

$$
\frac{\partial \tilde{p_{\|}}}{\partial \theta}+\left(\overline{p_{\perp}}-\overline{p_{\|}}\right) \epsilon \sin \theta=-\bar{n}_{w} q_{w} \frac{\partial \tilde{\Phi}}{\partial \theta}
$$

The $\theta$ - integration of $E q .(67)$ gives, using the relation $\tilde{p_{\|}} \simeq \tilde{n}_{w} k T_{\|}, \overline{p_{\|}} \simeq \bar{n}_{w} k T_{\|}$, and $\overline{p_{\perp}} \simeq \bar{n}_{w} k T_{\perp}$ based on the assumption that the temperature is almost constant along a magnetic field line due to large parallel heat conduction [25] $\left(\kappa_{\|} / \kappa_{\perp} \sim(\omega \tau)^{2} \gg 1\right)$,

$$
\frac{\tilde{n}_{w}}{\bar{n}_{w}}-\epsilon\left(\frac{T_{1}}{T_{\|}}-1\right) \cos \theta=-\frac{q_{w} \tilde{\Phi}}{k T_{\|}} .
$$

To eliminate $\tilde{n}_{w}$, we use the familiar Boltzmann relation for the isotropic background particles $\tilde{n}_{e} / \bar{n}_{\epsilon}=e \tilde{\Phi} / k T_{\epsilon}, \tilde{n}_{i} / \bar{n}_{i}=-Z_{i} e \tilde{\Phi} / k T_{i}, \tilde{n_{I}} / \overline{n_{i}}=-Z_{I} e \tilde{\Phi} / k T_{I}$, and the quasineutrality condition $q_{w} \tilde{n}_{w}=e\left(\tilde{n_{\epsilon}}-Z_{i} \tilde{n}_{i}-Z_{I} \tilde{n_{I}}\right)$. After some straightforward algebra, we get the equilibrium poloidal electrostatic potential variation as

$$
\frac{e \tilde{\Phi}}{k T_{e}}=\left[1+Z_{i}^{2} \frac{\bar{n}_{i}}{\bar{n}_{\epsilon}} \frac{T_{e}}{T_{i}}+Z_{I}^{2} \frac{\bar{n}_{I}}{\bar{n}_{\epsilon}} \frac{T_{\epsilon}}{T_{I}}+\frac{q_{w}^{2}}{e^{2}} \frac{\bar{n}_{w}}{\bar{n}_{e}} \frac{T_{\epsilon}}{T_{\|}}\right]^{-1} \epsilon \frac{q_{w}}{e} \frac{\bar{n}_{w}}{\bar{n}_{e}}\left(\frac{T_{\perp}}{T_{\|}}-1\right) \cos \theta
$$

Finally, using Eq. (52),

$$
\begin{gathered}
\frac{e \tilde{\Phi}}{k T_{e}}=\left[1+Z_{i} \frac{Z_{I}-Z_{e f f}}{Z_{I}-Z_{i}}\left\{1+\frac{Z_{I}}{Z_{i}} \frac{Z_{e f f}-Z_{i}}{Z_{I}-Z_{e f f}} \frac{T_{i}}{T_{I}}\right\} \frac{T_{e}}{T_{i}}+\frac{q_{w}^{2}}{e^{2}} \frac{\bar{n}_{w}}{\bar{n}_{e}} \frac{T_{e}}{T_{\|}}\right]^{-1} . \\
\cdot \epsilon \frac{q_{w}}{e} \frac{\bar{n}_{w}}{\bar{n}_{e}}\left(\frac{T_{\perp}}{T_{\|}}-1\right) \cos \theta .
\end{gathered}
$$

From $E q .(69)$, a few interesting points can be observed. First, if $\frac{q_{w}}{e} \frac{\bar{n}_{w}}{\bar{n}_{e}}\left(\frac{T_{\perp}}{T_{\|}}-1\right) \sim 1$ the poloidal potential variation can be of order $\epsilon$ as in Ref. [15], and is a function of temperature anisotropy. Second, the poloidal variation of electrostatic potential $\tilde{\Phi}$ is of $\cos \theta$ - type, originated from $\dot{B}$. Third, the sign of the potential is different for 
electron and ion cyclotron resonance heatings. For the ICRF heating case, $q_{w}>0$ and $T_{\perp} / T_{\|}>1$; and Eq. (69) yields $e \tilde{\Phi}>0$. For ECRH, $q_{w}<0$ and, $e \tilde{\Phi}<0$. This can be easily understood physically by the fact that the perpendicular heating of the ions (electrons) by ICRH (ECRH) traps excessive amount of positive (negative) charges at the smaller $B$ locations, thus the potential'inside the magnetic well should be positive (negative).

Assuming $\frac{q_{w}}{e} \frac{\bar{n}_{w}}{\bar{n}_{e}}\left(\frac{T_{1}}{T_{\|}}-1\right) \sim 1$, it can be observed that it is the thermal energy of colder background species that determines the potential depth. In the case of ECRH, the depth of the potential well is of the order of background ion thermal energy, if it is smaller than that of electrons. In the case of ICRH, the magnitude of $e \tilde{\Phi}$ variation may be roughly given by the electron thermal energy, if it is less than that of ions. In the case of mixed ECRH and ICRH, the sign of the potential variation will be determined by the dominance of the temperature anisotropy between the two species.

Equation (69) indicates that the poloidal potential is linearly dependent on the warm species density, and the calculation of the potential requires the knowledge of the density. In the case of minority ion heating, the minority ion density is given experimentally. Fig. 7 is the plot of the poloidal electrostatic potential generated by helium-3 minority heating in a deuterium plasma assuming the density of helium-3 is $10 \%$ of the electron density. As seen in the figure, the potential becomes no longer negligible in low $Z_{e f f}$ plasmas when $\bar{n}_{w} / \overline{n_{e}} \geq 0.1$.

If the warm species is indistinguishable with one of the cold species, however, it is not easy to determine the density of warm species. In this case, the density of warm species can be estimated either by experimental observations or by kinetic solution of the distribution function in the presence of cyclotron resonance heating. In ELMO Bumpy Torus [26], ECRH was used to produce a warm electron component and the density of warm electrons was measured to be $\leq 0.4$ of that of the cold electrons by soft x-ray diagnostics. Using a measured value of $\bar{n}_{w} / \bar{n}_{e}=0.3$ and an assumed value 
of $Z_{\text {eff }}=4$, then the normalized electric potential energy is approximately the inverse aspect ratio, $\epsilon$. 


\section{SUMMARY}

Temperature anisotropy of cyclotron resonance heated plasma species is studied and the resulting poloidal electrostatic potential variation is discussed. In order to estimate anisotropic temperatures, we solved perpendicular and parallel heat transport equations assuming negligible heat transport in the radial direction. A bi-Maxwellian distribution function is used for the warm particles and a full form of Coulomb collision operator is used to treat both ICRH and ECRH cases. In order to avoid numerical problems associated with singularity in bounce-averaged quantities at the trapped-passing boundary, analytic fitted forms are used as in Eq. (30).

By solving two simultaneous equations (36) and (37), it is found that for the ion cyclotron resonance case, the temperature anisotropy of the warm species can be as large as 15, as shown in Figure 5. With larger $Z_{\text {eff }}$, less temperature anisotropy was obtained since larger pitch angle scattering yields more effective spread of ion energy from the perpendicular to the parallel direction. Higher temperature anisotropy is seen with heavier mass ions. This is due to the mass effect in the drag term. More efficient tail heating can be expected with heavier mass for the same reason. In addition, either higher background temperature or smaller background density can produce higher anisotropy. For electron cyclotron resonance case, however, there exists a warm electron temperature run-away at $d_{e} \geq 0.61$ as shown in Figure 6 .

The poloidal electrostatic potential variation, $E q .(69)$, generated by the temperature anisotropy is calculated to be proportional to the inverse aspect ratio, ratio of warm and background densities, and temperature anisotropy. Since the temperature anisotropy can be as large as 15, the electrostatic potential becomes non-negligible when the density of warm species is more than $10 \%$ of that of the cold background species. If this is the case, it is important to include this potential in the transport and stability theory. 
In the future, we will extend the problem to higher wave power including, finite parallel wave number, hot resonant particles, relativistic correction in the quasilinear diffusion term, and loss mechanisms for the tail electrons.

\section{ACKNOWLEDGEMENTS}

This work was supported by the United States Department of Energy under Contracts Nos. DE-AC02-76-CH0-3073 and DE-FG02-86-ER53223. 


\section{References}

[1] HiNTON, F.L., HAZELTine, R.D., Rev. Mod. Phys. 48 (1976) 239.

[2] ROSEnBluth, M.N., HAZELTINE, R.D., Hinton, F.L., Phys. Fluids 15 (1972) 116.

[3] HAZELTINE, R.D., WARE, A.A. , Phys. Fluids 19 (1976) 1163.

[4] CHANG, C.S., HAZELTINE, R.D., Phys. Fluids 25 (1982) 536.

[5] CHANG, C.S., HAZELTINE, R.D., Nucl. Fusion 20 (1980) 1397.

[6] CHANG, C.S., HARVEY, R.W., Nucl. Fusion 23 (1983) 935.

[7] HSU, J.Y., CHAN, V.S., HARVEY, R.W., PRATER, R., WONG, S.K., Phys. Rev. Lett. 53 (1984) 564.

[8] CHAN, V.S., WONG, S.K.. Phys. Fluids 30 (1987) 830.

[9] TAGUCHI, M., J. Plasma. Physics, 47 (1992) 261.

[10] SHURYGIN, R.V., YUSHMANOV, P.N., Sov. J. Plasma Phys. 12 (1986) 306.

[11] CHANG, C.S., Phys. Fluids 26 (1983) 2140.

[12] STACEY, W.M. Jr., GTFR-63, Georgia Institute of Technology (1986).

[13] STRINGER, T.E., Phys. Fluids B 3 (1991) 981.

[14] TAGUCHI, M., Phys. Fluids B 4 (1992) 473.

[15] TAGUCHI, M., Plasma Phys. Control. Fusion, 35 (1993) 193.

[16] INDIRESHKUMAR, K., STACEY, W.M. Jr., Phys. Fluids B 5, 1850 (1993)

[17] OTT, E., MANHEIMER. W.M., Phys. Fluids, 19 (1976) 1035. 
[18] HINTON, F.L., Basic Plasma Physics I (ed. A.A. Galeev), p.163, North Holland, Amsterdam.

[19] KENNEL, C.F., ENGElmanN, F., Phys. Fluids 9 (1966) 2377.

[20] CHANG, C.S., LEE, J-Y., WEITZNER, H., Phys. Fluids B 3 (1991) 3429.

[21] STIX, T.H., Nucl. Fusion 15 (1975) 737.

[22] CHANG, C.S., COLESTOCK, P., Phys. Fluids B 2 (1990) 310.

[23] WOLFRAM, S., Mathematica. Second Edition, Addison-Wesley, 1991.

[24] CHEW, G.F., GOLdBerGer, M.L., LOW, F.E., Proc. Roy. Soc. London Ser. A236 (1956) 112.

[25] BRAGINSKiI, S.I., in Review of Plasma Physics Vol.1 (ed. M.A. Leontovich), p.228, Consultants Bureau, New York.

[26] SWAIN, D.W., COBBLE. J.A.. HILLIS, D.L., RICHARDS, R.K., UCKAN, T., Phys. Fluids 28 (1985) 1922. 

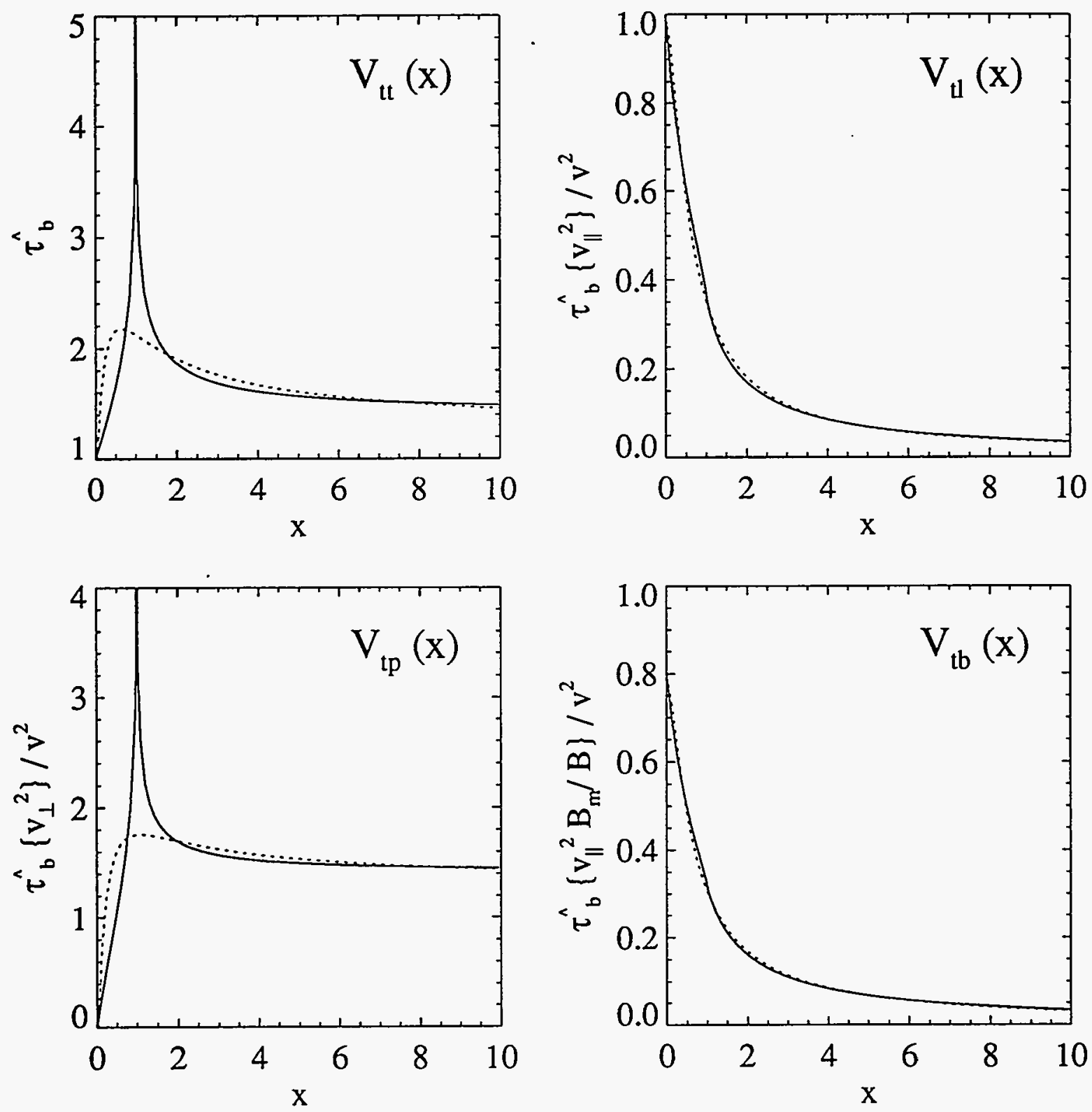

Figure 1: Solid lines show the bounced-averaged quantities in Eqs. (22)(25)(26)(29), which are to be integrated over $\lambda$. In order to avoid the divergence at $x=1$, the functions are fitted by polynomials of $\lambda$. 

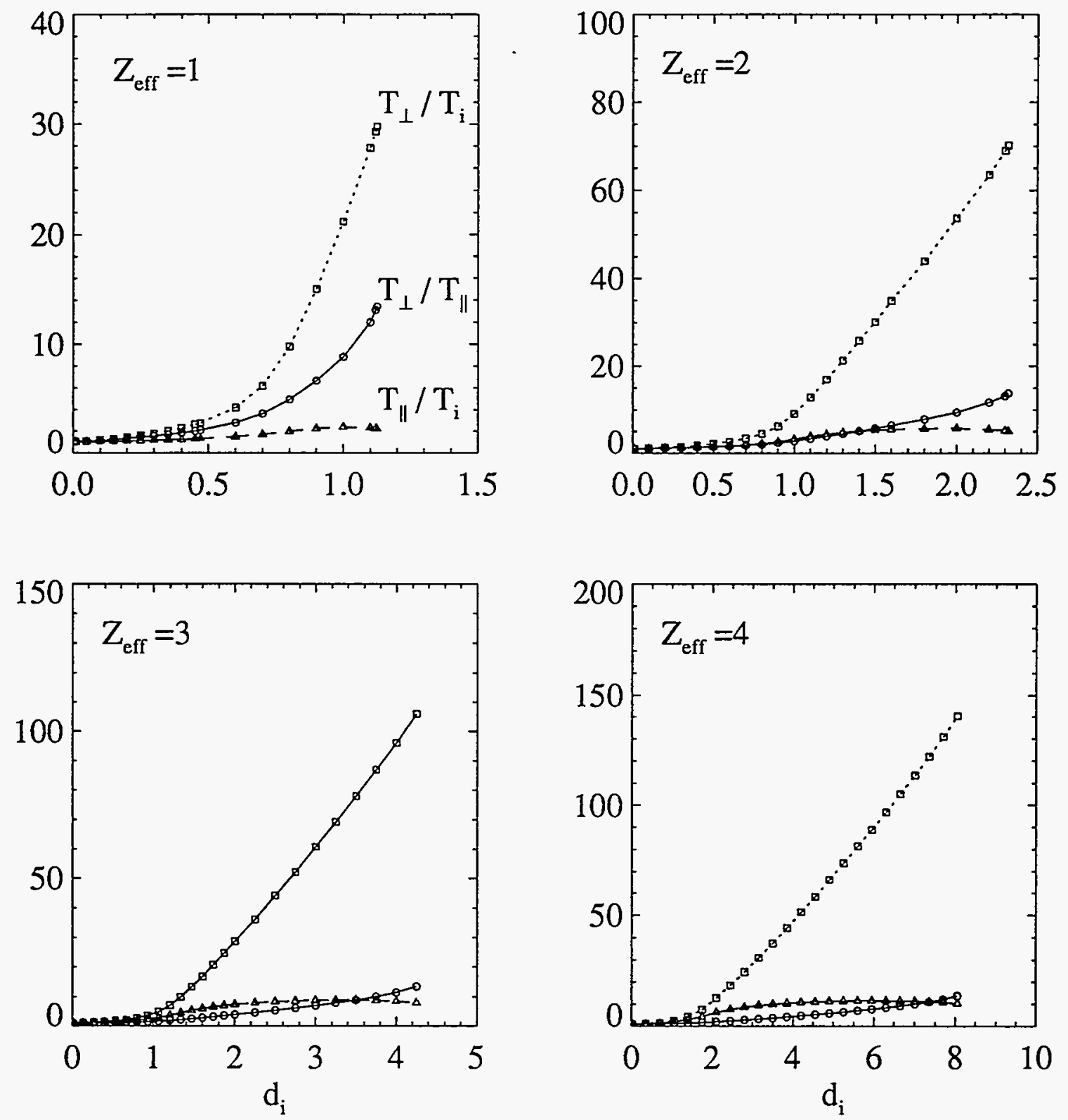

Figure 2: Anisotropic temperatures in the presence of ion cyclotron resonance heating in a hydrogen plasma with various $Z_{\text {eff }}$. For the calculation, a single impurity species (carbon) is included. $T_{i}$ is the background ion temperature and $d_{i}$ is the normalized input wave power. The preferential perpendicular heating of resonant ions by ICRF is shown. 

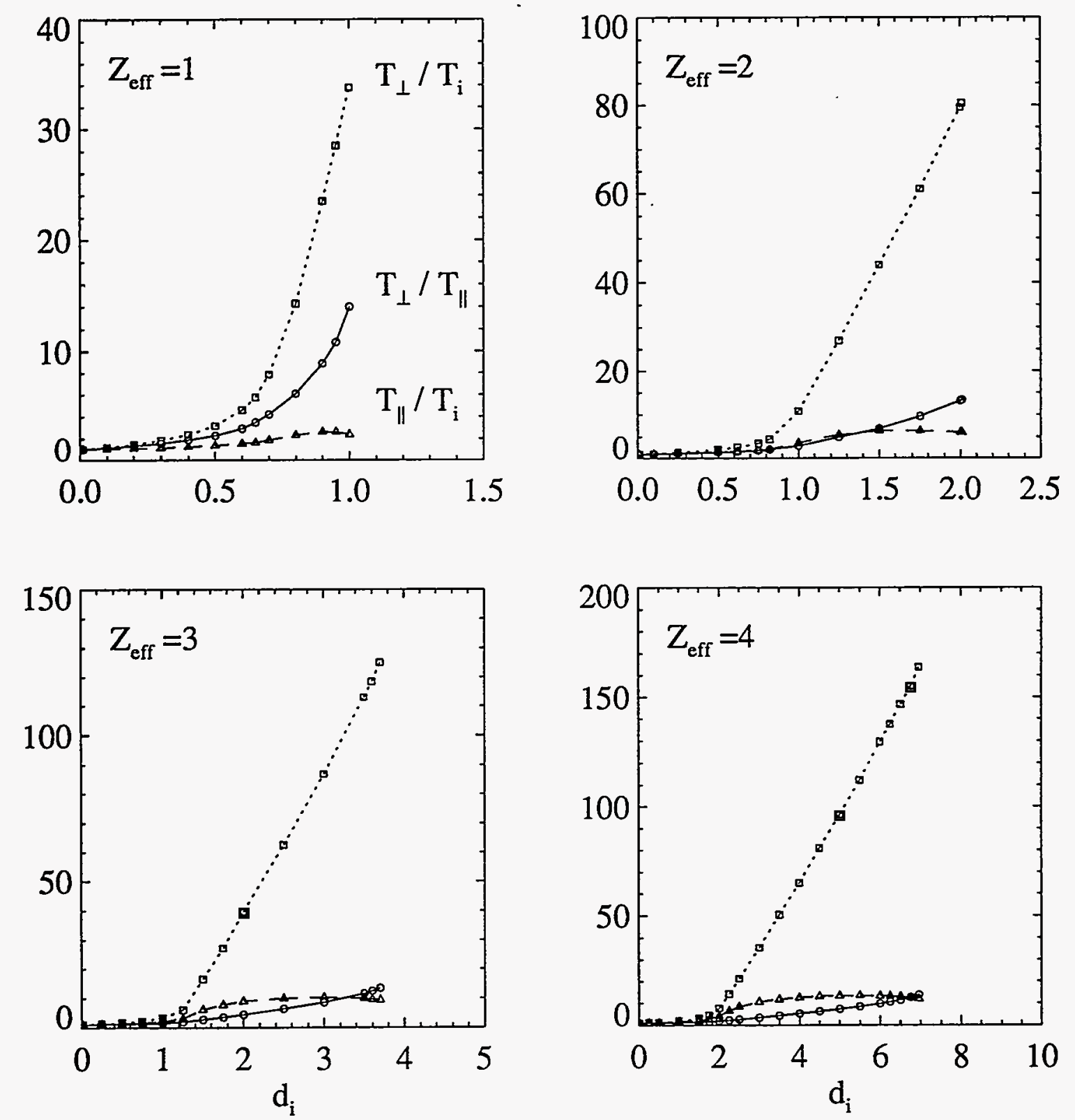

Figure 3: Anisotropic temperatures in the presence of ion cyclotron resonance heating in a deuterium plasma. 

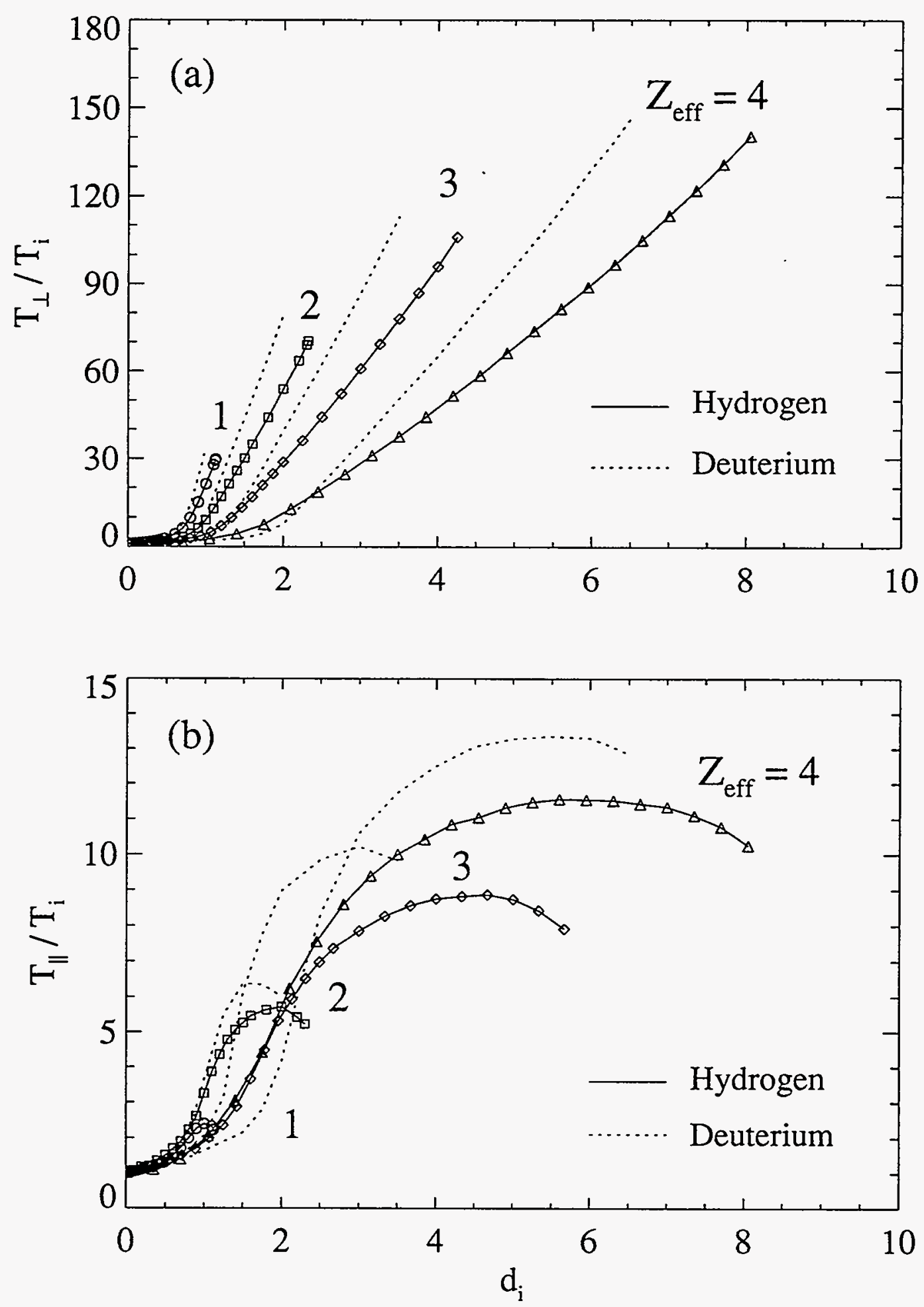

Figure 4: (a) The perpendicular and (b) the parallel temperature of warm ions. The parallel temperature reaches a maximum value and decreases with the input wave power. 


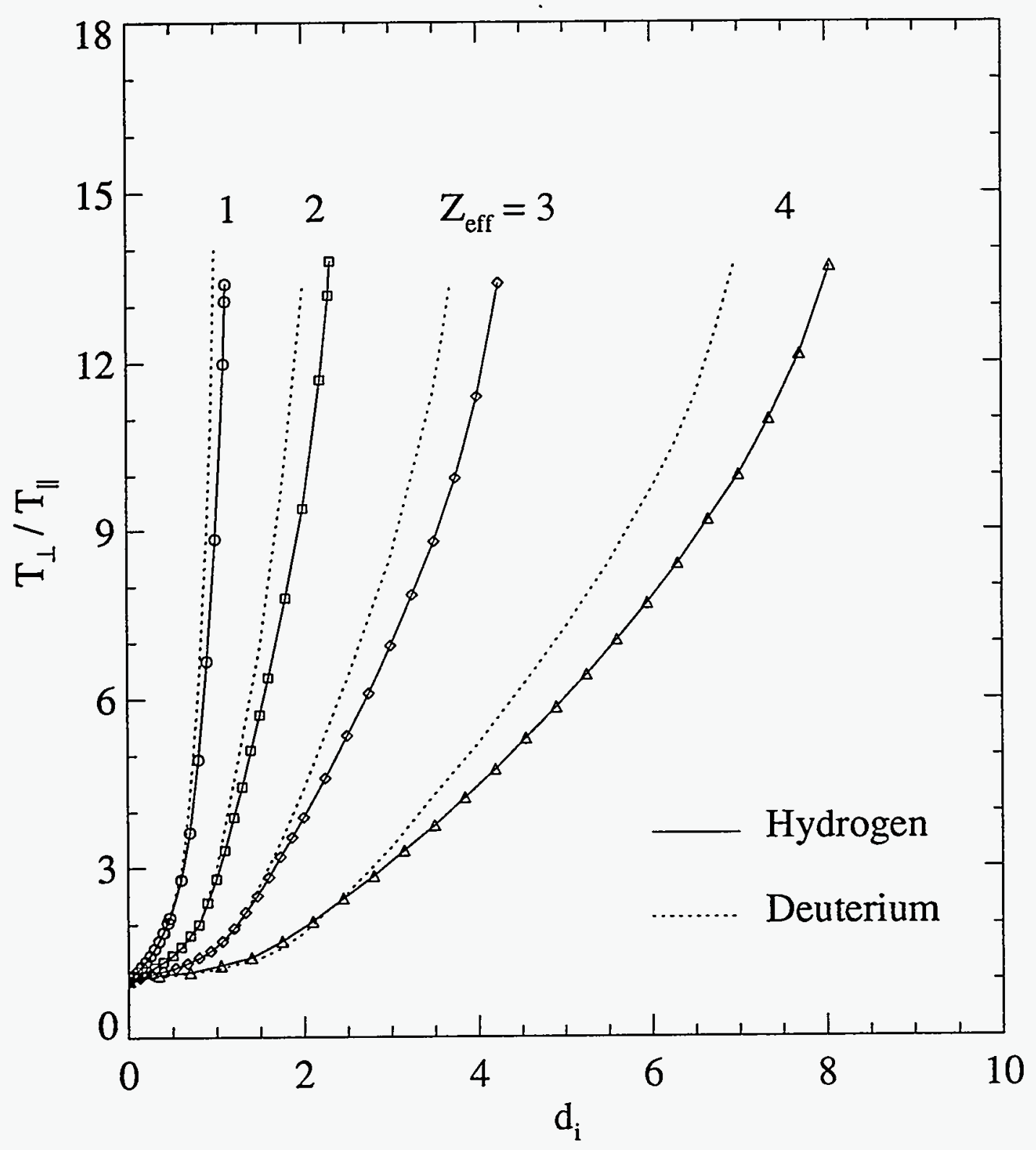

Figure 5: The temperature ratio of warm ions. 


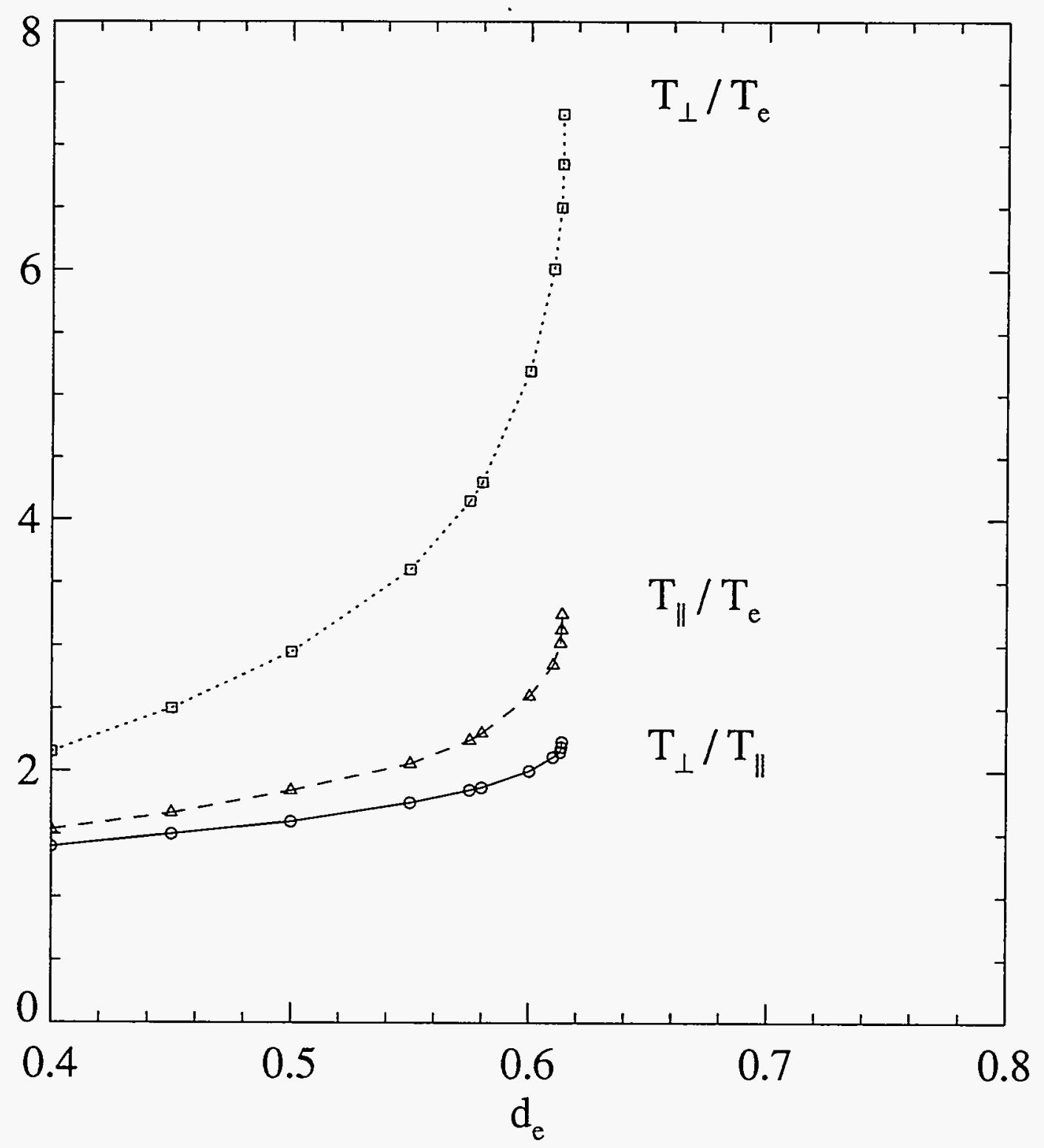

Figure 6: Temperature anisotropy by electron cyclotron resonance heating. Temperatures increase very rapidly at $d_{\epsilon} \simeq 0.61$ indicating runaway. $Z_{\text {eff }}=1$. 


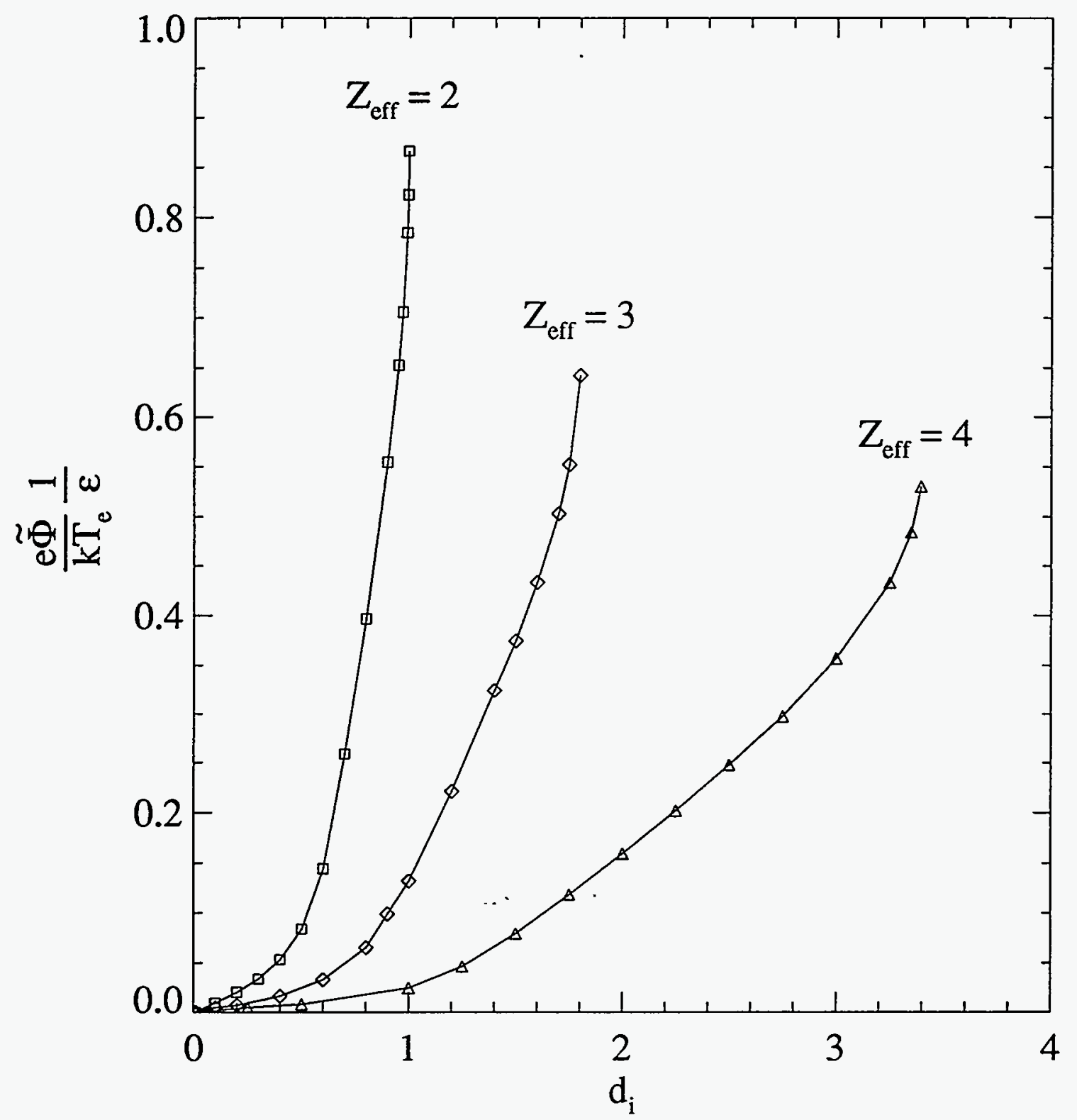

Figure 7: Poloidal electrostatic potential generated by helium-3 minority heating in a deuterium plasma. Density of helium-3 is assumed to be $10 \%$ of electron density. The background electron, ion, and carbon impurity temperature are assumed to be equal for simplicity. 


\section{EXTERNAL DISTRIBUTION IN ADDITION TO UC-420}

Dr. F. Paoloni, Univ. of Wollongong, AUSTRALIA

Prof. R.C. Cross, Univ. of Sydney, AUSTRALIA

Plasma Research Lab., Australian Nat. Univ., AUSTRALIA

Prof. I.R. Jones, Flinders Univ, AUSTRALIA

Prof. F. Cap, Inst. for Theoretical Physics, AUSTRIA

Prof. M. Heindler, Institut für Theoretische Physik, AUSTRIA .

Prof. M. Goossens, Astronomisch Instituut, BELGIUM

Ecole Royale Militaire, Lab. de Phy. Plasmas, BELGIUM

Commission-European, DG. XII-Fusion Prog., BELGIUM

Prof. R. Bouciqué, Rijksuniversiteit Gent, BELGIUM

Dr. P.H. Sakanaka, Instituto Fisica, BRAZIL

Prof. Dr. I.C. Nasćimento, Instituto Fisica, Sao Paulo, BRAZIL Instituto Nacional De Pesquisas Espaciais-INPE, BRAZIL

Documents Office, Atomic Energy of Canada Ltd., CANADA

Ms. M. Morin, CCFMTTokamak de Varennes, CANADA

Dr. M.P. Bachynski, MPB Technologies, Inc., CANADA

Dr. H.M. Skarsgard, Univ. of Saskatchewan, CANADA

Prof. J. Teichmann, Univ. of Montreal, CANADA

Prof. S.R. Sreenivasan, Univ. of Calgary, CANADA

Prof. T.W. Johnston, INRS-Energie, CANADA

Dr. R. Bolton, Centre canadien de fusion magnétique, CANADA

Dr. C.R. James, Univ. of Alberta, CANADA

Dr. P. Lukác, Komenského Universzita, CZECHO-SLOVAKIA

The Librarian, Culham Laboratory, ENGLAND

Library, R61, Rutherford Appleton Laboratory, ENGLAND

Mrs. S.A. Hutchinson, JET Library, ENGLAND

Dr. S.C. Sharma, Univ. of South Pacific, FIJI ISLANDS

P. Mähönen, Univ. of Helsinki, FINLAND

Prof. M.N. Bussac, Ecole Polytechnique,, FRANCE

C. Mouttet, Lab. de Physique des Milieux lonisés, FRANCE

J. Radet, CEN/CADARACHE - Bat 506, FRANCE

Prof. E. Economou, Univ. of Crete, GREECE

Ms. C. Rinni, Univ. of loannina, GREECE

Preprint Library, Hungarian Academy of Sci., HUNGARY

Dr. B. DasGupta, Saha Inst. of Nuclear Physics, INDIA

Dr. P. Kaw, Inst. for Plasma Research, INDIA

Dr. P. Rosenau, Israel Inst. of Technology, ISRAEL

Librarian, International Center for Theo Physics, ITALY

Miss C. De Palo, Associazione EURATOM-ENEA , ITALY

Dr. G. Grosso, Istituto di Fisica del Plasma, ITALY

Prof. G. Rostangni, Istituto Gas lonizzati Del Cnr, ITALY
Dr. H. Yamato, Toshiba Res \& Devel Center, JAPAN

Prof. I. Kawakami, Hiroshima Univ., JAPAN

Prof. K. Nishikawa, Hiroshima Univ., JAPAN

Librarian, Naka Fusion Research Establishment, JAERI, JAPAN

Director, Japan Atomic Energy Research Inst., JAPAN

Prof. S. Itoh, Kyushu Univ., JAPAN

Research Info. Crr., National Instit. for Fusion Science, JAPAN

Prof. S. Tanaka, Kyoto Univ.. JAPAN

Library, Kyoto Univ., JAPAN

Prof. N. Inoue, Univ. of Tokyo, JAPAN

Secretary, Plasma Section, Electrotechnical Lab., JAPAN

Dr. O. Mitarai, Kumamoto Inst. of Technology, JAPAN

Dr. G.S. Lee, Korea Basic Sci. Ctr., KOREA

J. Hyeon-Sook, Korea Atomic Energy Research Inst., KOREA

D.I. Choi, The Korea Adv. Inst. of Sci. \& Tech., KOREA

Prof. B.S. Liley, Univ. of Waikato, NEW ZEALAND

Inst of Physics, Chinese Acad Sci PEOPLE'S REP. OF CHINA

Library, Inst. of Plasma Physics, PEOPLE'S REP. OF CHINA

Tsinghua Univ. Library, PEOPLE'S REPUBLIC OF CHINA

Z. Li, S.W. Inst Physics, PEOPLE'S REPUBLIC OF CHINA

Prof. J.A.C. Cabral, Instituto Superior Tecnico, PORTUGAL

Prof. M.A. Heliberg, Univ. of Natal, S. AFRICA

Prof. D.E. Kim, Pohang Inst. of Sci. \& Tech., SO. KOAEA

Prof. C.I.E.M.A.T, Fusion Division Library, SPAIN

Dr. L. Stenflo, Univ. of UMEA, SWEDEN

Library, Royal Inst. of Technology, SWEDEN

Prof. H. Wilhelmson, Chalmers Univ. of Tech., SWEDEN

Centre Phys. Des Plasmas, Ecole Polytech, SWITZERLAND

Bibliotheek, Inst. Voor Plasma-Fysica, THE NETHERLANDS

Asst. Prof. Dr. S. Cakir, Middle East Tech. Univ., TURKEY

Dr. V.A. Glukhikh,Sci. Res. Inst. Electrophys.I Apparatus, USSR

Dr. D.D. Ryutov, Siberian Branch of Academy of Sci., USSR

Dr. G.A. Eliseev, I.V. Kurchatov Inst., USSR

Librarian, The Ukr.SSR Academy of Sciences, USSR

Dr. L.M. Kovrizhnykh, Inst. of General Physics, USSR

Kernforschungsanlage GmbH, Zentralbibliothek, W. GERMANY

Bibliothek, Inst. Fü Plasmaforschung, W. GERMANY

Prof. K. Schindler, Ruhr-Universitát Bochum, W. GERMANY

Dr. F. Wagner, (ASDEX), Max-Planck-Institut, W. GERMANY

Librarian, Max-Planck-Institut, W. GERMANY 